\title{
Investigación
}

\section{Prevalencia de Enfermedad Gingival en niños con Dentición Primaria.}

Gingival disease prevalence in

children with primary dentition.

Fecha de Recepción

I de junio de 20I I
Gengival prevalência da doença em

crianças com dentição decídua.
María Eugenia Martín Adscripta para perfeccionamiento en la Odontopediatría e iniciación en investigación.

Cátedra Odontopediatría.

\section{María Susana Discacciatti de Lértora \\ Directora. Profesora Adjunta \\ Cátedra Odontopediatría.}

Lugar de Trabajo

Cátedra Odontopediatría. Facultad de Odontología. UNNE. Avenida Libertad 5450.

Corrientes, Argentina.

\section{Resumen}

La enfermedad periodontal constituye una de las enfermedades de mayor frecuencia en la cavidad bucal, manifestándose especialmente como gingivitis en el paciente pediátrico. El propósito de este trabajo fue determinar la prevalencia de enfermedad gingival en niños/as con dentición primaria, concurrentes a la Cátedra de Odontopediatría de la FOUNNE en demanda de tratamientos odontológicos, durante el ciclo lectivo 2010. Previo consentimiento informado de padres/o responsables del niño/a. Fue un estudio transversal descriptivo, realizado en niños sanos, sin medicación general, con dentición primaria pura, evaluando el estado gingival por medio del Índice Gingival de Löe - Silness. Los resultados demostraron la presencia de enfermedad gingival en un $19,48 \%$, de la muestra, predominando en las niñas. La mayor frecuencia se presentó a los 5 años, incrementándose con la edad, en la población de niñas. En ambos sexos, solo se observó gingivitis leve en el 100\% de los casos.

Palabras claves

Gingivitis - infantes - salud gingival.

\section{Summary}

Periodontal disease is one of the most frequent diseases in the oral cavity, gingivitis especially as manifested in the pediatric patient. The purpose of this study was to determine the prevalence of gingival disease in children with primary dentition attending the Department of Dentistry 
of the FOUNNE in demand for dental treatment during the school year 2010. Informed consent from parents/or guardians of children. It was a descriptive study conducted in healthy children without medication generally pure primary dentition, gingival status evaluated by the Gingival Index of Löe - Silness. The results showed the presence of gum disease in $19.48 \%$ of the sample, predominantly in girls. Most cases occurred at age 5 increasing with age in the population of girls. In both sexes, only mild gingivitis was observed in $100 \%$ of cases.

\section{Keywords}

Gingivitis - Infants - gingival health.

\section{Resumo}

A doença periodontal é uma das doenças mais freqüentes na cavidade oral, gengivite especialmente manifestada no paciente pediátrico. $O$ objetivo deste estudo foi determinar a prevalência de doença gengival, em crianças com dentição decídua irão ao Departamento de Odontologia da FOUNNE na demanda por tratamento dentário durante o ano lectivo de 2010. O consentimento informado dos pais ou responsáveis de crianças. Foi um estudo descritivo, realizado em crianças com dentição saudável, sem medicamentos, geralmente pura primários, o estado gengival avaliada pelo Índice Gengival de LöeSilness. Os resultados mostraram a presença de doença periodontal em 19,48\% da amostra, com predominância nas meninas. A maioria dos casos ocorreu a cinco años, aumentando com a idade na população de meninas. Em ambos os sexos, apenas a gengivite leve foi observada em $100 \%$ dos casos.

\section{Palavras chave \\ Gengivite - saúde gengival - Crianças.}

\section{Introducción}

Estudios epidemiológicos realizados en innumerables países señalan que caries y enfermedad periodontal, son las afecciones de mayor prevalencia en la cavidad bucal, siendo ambas responsables de la pérdida de piezas dentarias. Jerarquizando las afecciones, las periodontopatías ocupan el segundo lugar, tanto por su prevalencia como por sus efectos.

Hoy se sabe que la enfermedad periodontal se inicia muy temprano en la vida del individuo, como se evidencia en los distintos tipos de gingivitis observada en niños. Se asegura que los niños que la padecen presentan una mayor predisposición a la enfermedad periodontal severa, cuando llegan a la edad adulta'. La incidencia va en aumento con la edad, asociada a deficiencias en la higiene bucal y a los cambios hormonales de la puber$\operatorname{tad}^{2}$. Estudios estadísticos realizados en EEUU indicaron una prevalencia de $45 \%$ a los 10 años, $67 \%$ a los 20 años y $70-80 \%$ a los 35 - 50 años, lo que demuestra que la enfermedad periodontal es evolutiva, pudiéndose inferir que, los niños de 10 años fueron portadores de gingivitis a los 7 - 8 años, suponiendo que la inflamación pudo haberse iniciado a edades tempranas en niños de 5 ○ 6 años con dentición primaria ${ }^{3}$.

La gingivitis del niño, no tiene el significado de la gingivitis del adulto. Se presume que existe una resistencia del huésped a presentar inflamación gingival durante los primeros años de vida, observándose que niños y adultos difieren en la propensión para desarrollar gingivitis ${ }^{4}$. En niños con dentición primaria, las características clínicas de la encía son diferentes a las del adulto. La gíngiva marginal presenta un aspecto más voluminoso, redondeado, estando relacionado con la pronunciada línea cervical de la corona de los dientes primarios. Presenta un color rosado intenso, debido a la presencia del epitelio escamoso estratificado más delgado y poco queratinizado, siendo la vascularización del corion más evidente ${ }^{5}$. El tejido conectivo es menos denso. La encía insertada no presenta puntillado en cascara de naranja en los primeros años, con un ancho variable de 1 a 6 $\mathrm{mm}$ que aumenta con la edad. El surco gingival es más profundo, alcanzando $2-5 \mathrm{~mm}^{6}$.

Cualquier desequilibrio entre los microorganismos del medio y los mecanismos de defensa del niño, provoca una respuesta inflamatoria gingival (gingivitis) que puede ser agravada por enfermedades sistémicas, particularmente las referidas al sistema inmune.

La gingivitis representa un espectro de enfermedades cuyo ataque normalmente se atribuye a la presencia de bacterias, habiendo además, otras formas de gingivitis que no se relaciona primariamente con placa bacteriana. Enfermedades sistémicas como la diabetes y leucemia, pue- 
den exacerbar la gingivitis asociada a placa, de igual modo que los cambios endocrinos, ciertas medicaciones y la desnutrición (deficiencia de vitamina $\mathrm{C}$ ). Las lesiones gingivales no inducidas por placa, pueden ser el resultado de patógenos bacterianos específicos como la Neisseria, como también pueden deberse a infecciones virales e infecciones fúngicas ${ }^{7}$. La Academia Americana de Periodoncia (AAP) incluye categorías para todas estas formas de gingivitis ${ }^{8}$ que afectan solamente el periodonto de protección (gíngiva), pudiendo ser crónica, aguda, localizada o generalizada. Tanto para los niños de dentición primaria como para los adolescentes, las características clínicas son semejantes, siendo su principal forma de presentación, la enfermedad gingival inducida por placa bacteriana'. Esta afección, por lo general pasa sus estadios iniciales sin sintomatología. Suele aparecer en la infancia y por no ser diagnosticada ni tratada, evoluciona a lesiones complejas en el adulto, llegando a ser la causa de extracciones dentales masivas después de los 35 años, debido a la periodontitis alcanzada². La patología comienza con una inflamación del margen gingival, que se vuelve crónica, pudiendo llegar hasta la encía insertada $^{10}$ ostentando como signo de inflamación relevante, el edema y cambio de coloración gingival, virando a un tono más intenso, sin pérdida de inserción o hueso alveolar.

La gingivitis crónica, es la más común en niños y adolescentes, causada por factores locales, especialmente placa bacteriana. Desde los estudios de Loe y colaboradores, se conoce que la acumulación de bacterias sobre el margen gingival produce una reacción inflamatoria"'. La placa aumenta cuantitativamente y varía cualitativamente a medida que el niño crece. En la placa de niños con gingivitis, a los 7 años ya se pueden encontrar microorganismos anaerobios y espiroquetas. En un estudio realizado en infantes con y sin sangrado gingival, se describe la presencia de valores de microorganismos compatibles con enfermedad al ser explorado el surco gingival de piezas dentarias temporarias $^{12}$.La placa dentobacteriana y la microbiota del surco gingival constituyen el factor de riesgo más fuertemente asociado con el origen y la evolución ulterior de la gingivitis causada por el contacto mantenido con la encía y como resultado de un hábito incorrecto de higiene bucal ${ }^{2}$.

Numerosos autores aseveran la presencia de gran cantidad de placa bacteriana en niños ${ }^{13}$ además de describir la importancia de un diagnostico precoz y aplicación de medidas preventivas para evitar el avance de la enfermedad hasta la edad adulta, época en que se agrava, pudiendo llevar a la perdida de piezas dentarias.

En el niño sano, por lo general, a pesar de la elevada prevalencia de gingivitis, no suele observarse la típica evolución de gingivitis a periodontitis, como ocurre en los adultos, por lo que la incidencia de formas crónicas y agresivas de la enfermedad periodontal es baja. Estas últimas suelen estar asociadas con enfermedades sistémicas ${ }^{10}$.

El propósito del presente trabajo es evaluar el estado de salud gingival en niños con dentición primaria, a fin de conocer la epidemiología zonal a edades tempranas, y motivar a odontopediatras, ortodoncistas y odontólogos generales que atiende niños y adolescentes, a reconocer signos mínimos de enfermedad gingival para poder prevenir, diagnosticar precozmente y tratar o derivar oportunamente, para poder evitar severas periodontitis del adulto, originadas en gingivitis no diagnosticadas ni tratadas durante la infancia.

\section{Objetivo General}

Determinar la prevalencia y severidad de enfermedad gingival, en una población de niños/as de la ciudad de Corrientes, que presentan dentición primaria,

\section{Objetivos Específicos}

a.Observar clínicamente el estado de salud gingival en los niños/as de la muestra.

b.Aplicar el Índice Gingival de Löe- Silnes, según dientes parámetros.

c.Personalizar al paciente según Actividad Gingival.

d.Determinar grado de severidad gingival predominante según edad y género

e.Analizar estadísticamente los resultados obtenidos.

\section{Materiales y Método}

Se examinaron los niños/as concurrentes a la Clínica de Odontopediatría de la FOUNNE durante el ciclo lectivo 2010. Previo consentimiento informado de los padres o responsables 
de los mismos, se realizó el examen de los niños/ as, aplicando los siguientes criterios de inclusión: niño/a sano, con dentición primaria pura, sin tratamiento farmacológico de ningún tipo, obteniéndose una muestra conformada por 77 niños/as (40 niñas y 37 varones).

A fin de establecer la actividad y severidad de la enfermedad gingival, se utilizó el Índice cualitativo de Loe- Sillness ${ }^{14}$ considerando como dientes parámetros las piezas 5.5, 6.I , 6.4, 7.5, 8.I, 8.4.

Confección del Índice: Previa motivación, se posicionó correctamente al niño, sentado en el sillón dental procediendo a evaluar las encías (Figura.I). En niños, el sondeo del surco gingival resulta incómodo y doloroso y complica el abordaje psicológico, por lo que en esta investigación, no se utilizó sonda, limitando el examen a la inspección visual, de los tejidos gingivales (que permitan identificar cambios en el color y el contorno, así como signos de inflamación) utilizando instrumental de inspección de rutina y buena luz. Para la observación clínica, se dividió la superficie gingival de cada diente parámetro en 4 zonas: mesial, central, distal (vestibulares) y palatino-lingual (palatino-lingual) como demuestra la Figura 2 , procediéndose luego al secado de las superficies gingivales, palpación de la gíngiva marginal, presionando suavemente con el dedo índice la zona a evaluar y valoración del estado gingival, de acuerdo al siguiente criterio:

0: Color rosa pálido. Consistencia firme.

I: Ligero cambio de color. Edema. Sin sangrado.

2: Marcado cambio de color. Edema. Sangrado a la presión que desaparece al cesar el estímulo.

3: Tejidos gingivales muy enrojecidos. Edema neto. Hemorragia espontánea.

Los grados obtenidos fueron volcados a una Tabla de Registro (Figura 3) ubicando cada valor en el casillero correspondiente a los dientes parámetros y superficies gingivales examinadas: mesiovestibular (M), central (C), disto-vestibular (D) y palatino-lingual $(\mathrm{P})$. Hallando la media de los valores asignados en diente evaluado. Finalmente se obtuvo el promedio de los 6 dientes parámetros, lográndose como resultado un número absoluto indicador del estado gingival del niño evaluado (Valor del Índice).

Interpretación de los valores del Índice: Valor menor a uno $(<1)=$ Compatible con salud. Encía Normal $(\mathrm{N})$. Valor mayor a uno $(>\mathrm{I})=$
Actividad o enfermedad gingival (AG), clasificada según su valor en: Inflamación Leve (I); Inflamación Moderada (2); Inflamación Grave (3).

\section{Resultados}

Se examinaron 77 pacientes con dentición primaria $(51,9 \%$ niñas y $48 \%$ varones) de edades entre 4 y 6 años, distribuidos según género como se observa en la Tabla I.

En 15 casos de la muestra (19\%) se observó Enfermedad Gingival y el resto (81\%) presentó valores compatibles con salud (Figura 4). En relación a los grupos etarios, la mayor frecuencia de gingivitis se observó a los 5 años (73.3\%), registrándose los menores valores a los 4 años (6.6\%), tal como describe la Figura 5.

En el $100 \%$ de los casos, los niños/as reportaron Índice de Loe - Silness Grado I, determinante de gingivitis leve, sin obtener registros de Índices 2 y 3 (Figura 6).

En cuanto a la discriminación por sexo, en las Niñas, los resultados demostraron un $77,5 \%$ de salud gingival y $22,5 \%$ de gingivitis leve, manifestándose la enfermedad en todas las edades estudiadas (Tabla II), siendo la prevalencia directamente proporcional a la edad, reportando a los 4 años: I I, I\%, a los 5 años: 24, I3\% y a los 6 años: $50 \%$ (Figura 7).

En la población de Varones, los resultados demostraron $83,7 \%$ compatible con salud gingival y 16 , $2 \%$ con gingivitis de grado leve, sin hallazgos de inflamación moderada o severa. La Tabla III detaIla la presencia de enfermedad gingival a los 5 y 6 años, resultando la prevalencia inversamente proporcional a la edad, ostentando un $25 \%$ a los 5 años y $16.6 \%$ a los 6 (Figura 7).

Comparando ambas poblaciones, la Figura 7 demuestra que la enfermedad gingival en las niñas se presenta desde los 4 años, igualando la frecuencia con los varones a los 5 años, para luego aumentar y sobrepasarlos a los 6 años. En tanto los Varones, reportaron enfermedad gingival a partir de los 5 años, disminuyendo la prevalencia a los 6.

La prevalencia en Niñas, es mayor que en los Varones.

\section{Discusión}

Los resultados del presente estudio demostra- 
Tabla I. Distribución porcentual de la población, según edad y género.

\begin{tabular}{|l|c|c|c|c|}
\hline \multirow{2}{*}{ EDAD } & \multicolumn{2}{|c|}{ FEMENINO } & \multicolumn{2}{c|}{ MASCULINO } \\
\cline { 2 - 5 } & Total & Porcentaje & Total & Porcentaje \\
\hline 4 años & 9 & $22,5 \%$ & 9 & $24,3 \%$ \\
\hline 5 años & 29 & $72,5 \%$ & 16 & $23,2 \%$ \\
\hline 6 años & 2 & $5 \%$ & 12 & $32,4 \%$ \\
\hline Totales & 40 & $100 \%$ & 37 & $100 \%$ \\
\hline
\end{tabular}

Tabla 2. Distribución de niñas con Enfermedad Gingival según la edad.

\begin{tabular}{|l|c|c|}
\hline EDAD & $\begin{array}{c}\mathrm{N}^{\circ} \text { de Niñas } \\
\text { examinadas }\end{array}$ & $\begin{array}{c}\mathrm{N}^{\circ} \text { de casos con } \\
\text { enfermedad gingival }\end{array}$ \\
\hline 4 años & 9 & 1 \\
\hline 5 años & 29 & 7 \\
\hline 6 años & 2 & 1 \\
\hline Total & 40 & 9 \\
\hline
\end{tabular}

Tabla 3. Distribución de varones con Enfermedad Gingival según la edad.

\begin{tabular}{|l|c|c|}
\hline EDAD & $\begin{array}{c}\mathrm{N}^{\circ} \text { de Varones } \\
\text { examinados }\end{array}$ & $\begin{array}{c}\mathrm{N}^{\circ} \text { de casos con } \\
\text { enfermedad gingival }\end{array}$ \\
\hline 4 años & 9 & 0 \\
\hline 5 años & 16 & 4 \\
\hline 6 años & 12 & 2 \\
\hline Total & 37 & 6 \\
\hline
\end{tabular}

Figura I. Observación clínica de la gíngiva en dentición primaria.

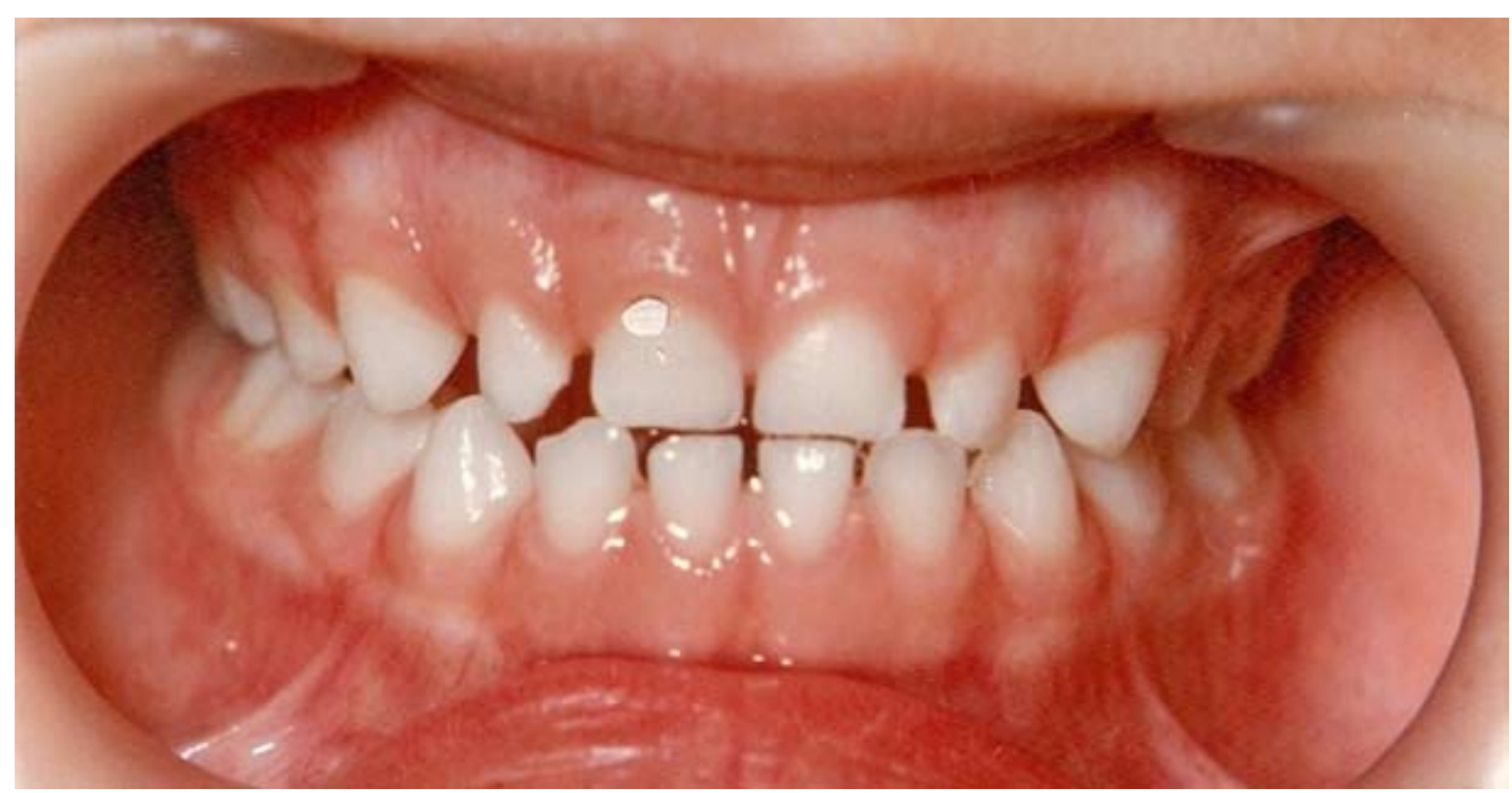

Figura 2. Zonas de observación clínica para el Índice de Löe y Silness.
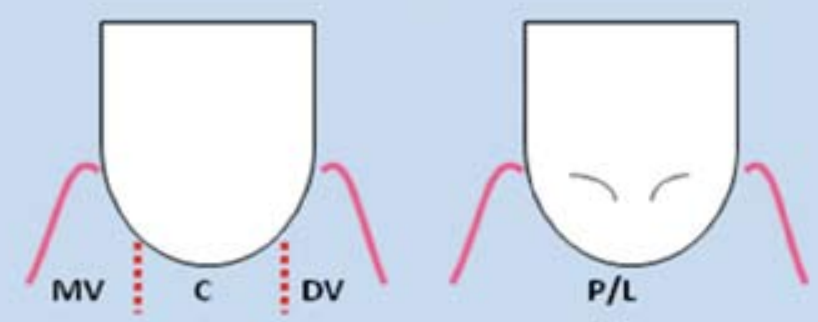
Figura 3. Tabla de Registro del Índice Gingival de Löe y Silness.

\begin{tabular}{|c|c|c|c|c|c|}
\hline $\begin{array}{c}\text { Dientes } \\
\text { Parámetros }\end{array}$ & $\begin{array}{c}\text { Mesio } \\
\text { Vestibular. }\end{array}$ & Central & $\begin{array}{c}\text { Disto } \\
\text { Vestibular }\end{array}$ & $\begin{array}{c}\text { Palatino } \\
\text { Lingual }\end{array}$ & $\begin{array}{c}\text { Valor } \\
\text { Promedio }\end{array}$ \\
\hline \multicolumn{6}{|l|}{5.5} \\
\hline \multicolumn{6}{|l|}{6.1} \\
\hline \multicolumn{6}{|l|}{6.4} \\
\hline \multicolumn{6}{|l|}{7.5} \\
\hline \multicolumn{6}{|l|}{8.1} \\
\hline \multicolumn{6}{|l|}{8.4} \\
\hline & & & & Valor: & \\
\hline
\end{tabular}

Figura 4. Prevalencia de enfermedad gingival en niños con dentición primaria.

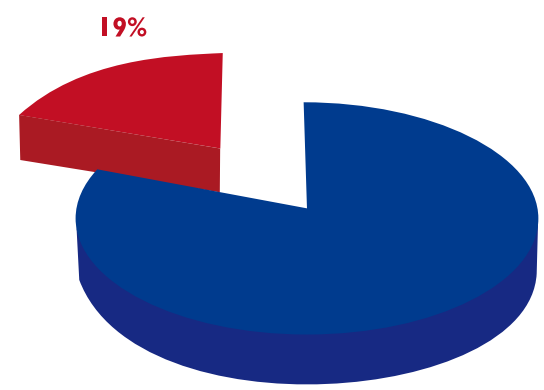

$81 \%$

Sin actividad gingival

Con actividad gingival

Figura 5. Frecuencia de enfermedad gingival según edades.

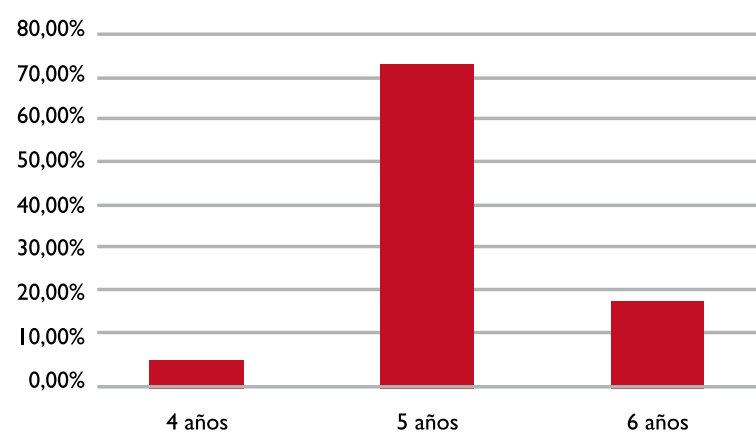

ron que el $81 \%$ de los niños examinados presentaban valores compatibles con salud gingival, cifra aproximada al $79 \%$ reportado por Dho y col ${ }^{(15)}$. En relación a la enfermedad gingival se registró un $19 \%$ de actividad, concordando con los hallazgos de Marquillas Bras ${ }^{(1)}$, quién describió $18 \%$ de gingivitis en niños de 5 años en el Reino Unido, y Rodríguez Carrasco y col. ${ }^{(16)}$, que demostró $19.7 \%$ de menores afectados con enfermedad gingival y $80.2 \%$ libres de esta patología.

Los valores hallados ratifican las conclusiones de Marín ${ }^{(5)}$ respecto a la baja tendencia a desarrollar gingivitis en la dentición primaria, pero difieren de los datos aportados por otros investigadores que observaron mayor actividad gingival. Así Lescano de Ferrer ${ }^{(17)}$, en un análisis de 80 niños en Córdoba, Argentina, informó un 35\% de gingivitis en dentición temporaria, sin antecedentes de salud general en el $77 \%$ de los casos, presentando niveles leve y moderado; Jahn y col. ${ }^{(13)}$, demostró que niños de I - 5 años de edad de clase social baja, presentaban $84,3 \%$ de gingivitis y $53 \%$ de hemorragia a la presión, situación que podría estar relacionada con el bajo nivel socio económico de la población, lo que actúa como factor de riesgo importante de contraer enfermedad gingival, debido a la falta de prevención y de atención odontológica y al estado nutricional deficiente, dato ratificado por las investigaciones de Olivera García y col. ${ }^{(2)}$ quien en un estudio realizado en La Habana, describió $83.3 \%$ de enfermedad gingival en escolares de 6 a 14 años, presentando la mayoría (86\%) índices gingivales leves, destacando alta prevalencia de gingivitis en la población estudiada, pero bajos valores del Índice Gingival de 
Löe y Silness. Por su parte, Norman Harris ${ }^{(18)}$, en el libro "Odontología Preventiva Primaria" divulgó datos epidemiológicos de gingivitis en infantes de Islandia, reportando $39 \%$ de gingivitis y $16 \%$ de sangrado gingival en una población de 230 niños de 6 años de edad y Pauritaite y col. ${ }^{(19)}$ en 250 niños con dentición temporaria y mixta temprana, halló $47.2 \%$ de gingivitis leve, todos valores superiores a los hallazgos del presente estudio.

Numerosos estudios expusieron resultados de alta prevalencia de enfermedad gingival: Juárez López y col ${ }^{(20)}$, demostraron en pre-escolares alteraciones periodontales en un $70 \%$; Franco ${ }^{(21)}$, señala una alta prevalencia de gingivitis en dentición primaria (7I,I\%), Benítez Melo y col.(9) reportaron $90 \%$ de gingivitis incipiente en niños de Bogotá, mencionando que el grupo de 3 - 6 años presenta valores menores en el índice gingival, en relación a edades mayores.

En relación a la aplicación del índice, González y col. ${ }^{(22)}$ en un estudio realizado en niños entre 2 y 5 años de edad, observó que el índice gingival basado en la presencia o no de sangrado gingival, no es un índice muy confiable en niños para detectar cambios en relación a una buena higiene oral, debido a que cuando los niños presentan enfermedades sistémicas tales como gripe y enfermedades infecciosas, entre otras, el nivel de higiene oral decrece, permitiendo que en la medición del índice gingival, se observe un aumento.

En el año 2000, la Organización Panamericana de la Salud (OPS) y la Organización Mundial de la Salud (OMS) en el artículo "La salud de la niñez"(23) reportaron que $50 \%$ de los niños de países de América, entre 5 y 14 años, padecen enfermedades gingivales. Así mismo, la OMS en el año 2004, en el artículo "La OMS publica un nuevo informe sobre el problema mundial de las enfermedades buco dentales"(24) afirmó que la mayoría de los niños del mundo presentan signos de gingivitis. "Epidemiology of Periodontal Diseases"(25) publicado en 2005 refiere una alta prevalencia de gingivitis en niños de Estados Unidos, con valores superiores al $40 \%$ - $60 \%$ de escolares afectados, en tanto Coutinho y col $^{(26)}$, señalan una prevalencia de $83,3 \%$ de enfermedad gingival en infantes de 4 a 12 años, coincidiendo con datos de alta prevalencia entre las edades de 6 a 8 años aportados por Varas ${ }^{(27)}$ en su análisis estadístico sobre el estado periodontal en niños de la región
Figura 6. Frecuencia de enfermedad gingival, según grado de severidad.

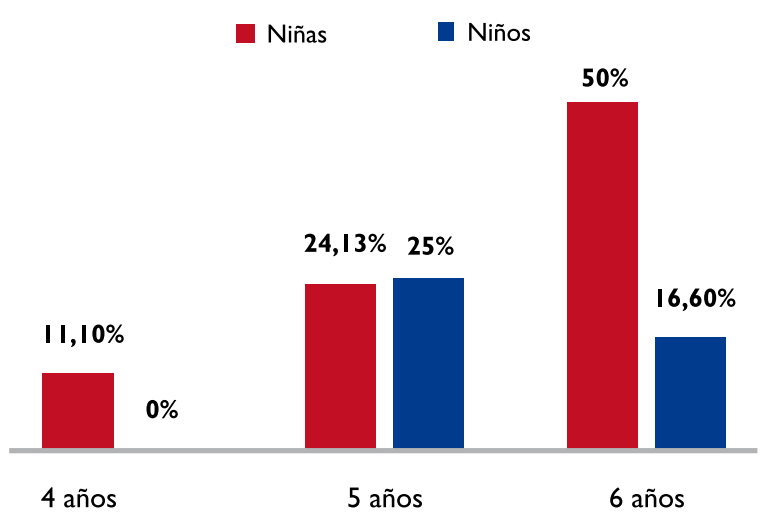

Figura 7. Prevalencia de gingivitis según edad y sexo.

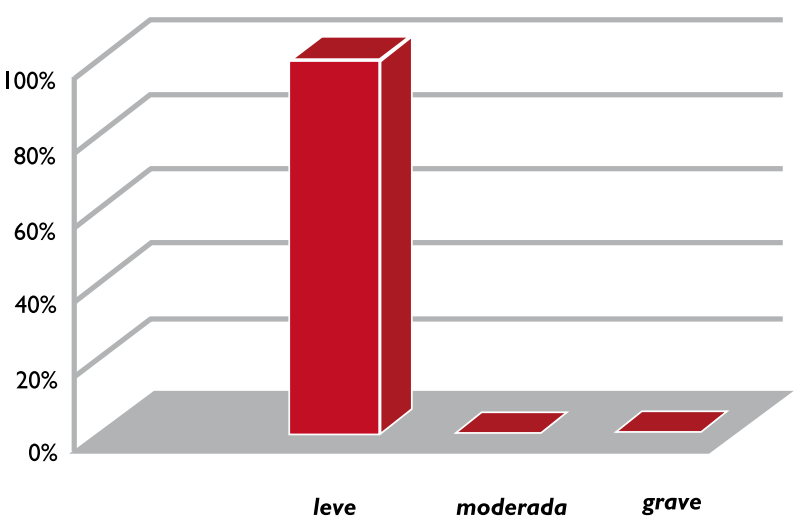

metropolitana de Chile. Es importante señalar, que estos últimos trabajos, abarcan edades analizadas en nuestra investigación ( 4 a 6 años) y edades correspondientes a la dentición mixta.

En relación al género, el número de varones estudiado según grupo etario, se distribuyó de manera más equilibrada en comparación a las niñas, en las que solo existieron 2 casos de 6 años y la gran mayoría se concentró a la edad de 5 años.

Coincidiendo con lo informado por Varas y col $^{(27)}$, en esta investigación se encontró una diferencia porcentual en la actividad gingival entre los géneros, siendo las niñas más afectadas (22,5\%), en relación a los varones (16,21\%), ratificando lo reportado por Dhar ${ }^{(28)}$.

Respecto a la edad de inicio, Vila y col. ${ }^{(29)}$ opinaron que esta enfermedad se inicia a los 5 años. En este estudio, se registraron casos positivos a los 4 años de edad, aunque el pico más alto de Actividad Gingival correspondió al grupo de 
5 años. Según Juárez - López y col(20) los 5 años, constituyen una etapa de transición de las denticiones, que provoca inflamación gingival debido a ligeros traumatismos al masticar y por falta de cepillado, motivado por la hipersensibilidad de las piezas dentarias próximas a exfoliarse, contrario a las conclusiones de Franco ${ }^{(21)}$, quien expresa que las edades con mayor prevalencia están comprendidas entre los 2 y 3 años por la presencia de factores de riesgo: uso de biberón, pobre higiene oral, cambios en los hábitos alimentarios.

En cuanto a la evolución, numerosas investigaciones demuestran que la gingivitis comienza en la infancia y la incidencia y grado de severidad, se incrementa hacia la adolescencia. La prevalencia hallada demostró un incremento relacionado con la edad, coincidiendo con Ketabi y col $^{(30)}$ quien indicó una tendencia de aumento de la severidad de esta enfermedad, proporcional al aumento de la edad. Por su parte, Rodríguez-Carrasco ${ }^{(16)}$ registró $5 \%$ de casos a los 3 años y $50 \%$ a los 6 años, demostrando un claro incremento con los años, coincidiendo con varios investigadores: Benítez Melo y col. ${ }^{(9)}$, Juárez - López y col. ${ }^{(20)}$, Pauritaite y coll $^{(19)}$, Varas y col. ${ }^{(28)}$, quienes afirman que existe mayor predisposición a desarrollar gingivitis a medida que aumenta la edad debido a una mayor respuesta gingival a la placa bacteriana.

En relación a la severidad de la gingivitis, puede expresarse, que en el niño sano no es frecuente la aparición de formas serias de gingivitis o periodontitis. A pesar de la elevada incidencia de gingivitis las manifestaciones de enfermedad gingival más severas se asocian a enfermedades sistémicas ${ }^{(10)}$ La severidad de la enfermedad gingival hallada en ambos grupos correspondió a un grado de gingivitis leve, con ausencia de grados moderados o severos de la enfermedad, coincidiendo con los hallazgos de Murrieta Pruneda y col. ${ }^{(31)}$ Benítez Melo(9) afirma que la severidad de la gingivitis es menos intensa en niños que en individuos mayores con similares cantidades de placa bacteriana. Esto resulta cierto al comprobar la ausencia de gingivitis moderada o severa en niños afectados al presente trabajo, si se tiene en cuenta que, según datos obtenidos de la Historia Clínica, el 100\% de la muestra eran pacientes de alto riesgo con valores de O'Leary mayores a $20 \%$. La gingivitis leve hallada en los niños examinados, coincide con hallazgos de Olivera García( ${ }^{(2)}$, Bustamante

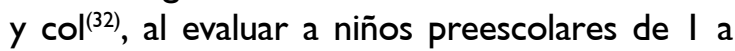

5 años con el índice de Löe - Silness y con lo demostrado por autores como Pauritaite ${ }^{(19)}$, Juárez - López ${ }^{(20)}$ y Rodríguez - Carrasco ${ }^{(16)}$ quienes publicaron sobre la prevalencia de gingivitis de grado leve en dentición temporaria, con solo $0,4 \%$ de gingivitis moderada y ausencia de tipo severa.

Los resultados de esta investigación corroboran los dichos de Lindhe ${ }^{(33)}$ quien asegura que la menor cantidad de patógenos en la placa bacteriana, las características morfológicas (mayor irrigación del tejido conjuntivo) y mayor aporte de sistema inmunitario con una respuesta inflamatoria de menor intensidad, son factores relacionados con la baja prevalencia y gravedad de gingivitis observada en niños con dentición temporaria pura.

\section{Conclusiones}

I.En la muestra estudiada, la prevalencia de gingivitis en dentición primaria es de 19,4\%, predominando en las niñas.

2.En ambos sexos, solo se observó gingivitis leve, en el $100 \%$ de la muestra.

3.La mayor prevalencia se presentó a los 5 años, incrementando con la edad en la población de niñas.

4.Estos hallazgos determinan que en niños con dentición primaria, el examen de los tejidos periodontales debe estar presente en las consultas de rutina, para establecer diagnósticos tempranos, brindar tratamientos oportunos y prevenir en consecuencia patologías mayores. 


\section{Bibliografía}

I. Jaramillo, D. C. Odontología Pediátrica. Fundamentos de Odontologia. 3ra ed. Bogotá, Colombia: Corporación para investigaciones biológicas; 2003.

2. Olivera García M. L., Iglesias Berlanga I. J. Enfermedad Periodontal e Higiene Bucal en escolares. Revista de Ciencias Médicas La Habana 2009. [Acceso: 15 de abril de 2010]; 15(I).Disponible en: http://www.cpicmha.sld.cu/hab/voll5_I_09/ habl2109.html

3. Sarian R, Cesario A. D, Castro J.C: doencas peridontais na infancia e adolescencia en Guedes Pintos A. Odontopediatria., Sao Pablo, Brasil. Ed. Santos editora Ltda. 7ma año 2003. Pág. 325 353.

4. Matsson L. Development of gingivitis pre - school Children and young adults. Journal of Clinical Periodontology 14 Dec 2005. [Acceso: 20 de abril de 2010]; 5 (I) [24-34]. Disponibleen:http://www3. interscience.wiley.com/journal// | 96 |8703/abstract? CRETRY $=\mid$ \&SRETRY $=0$

5. Marin R. H. Estudio comparativo de las características histológicas de encías clínicamente normales e inflamadas en niños. Resumen M-02I. Reunión de Comunicaciones Científicas de SGCyT UNNE. Año 2003. Disponible en: http:// wwwl.unne.edu.ar/cyt/2003/comunicaciones/03Medicas/M-02I.pdf.

6. Carranza F. A. Periodontología Clínica de Glickman 6 ed. México. Ed. Interamiericana: 1986: 330.

7. García Linares S: Nueva Clasificación de la Enfermedad Periodontal. Odontología Sanmarquina 2003; 6 (II): 48 - 50.

8. Olarte C, Ortega C.J. Enfermedad Peridontal: Una nueva clasificación. Revista de Odontología. Disponible enhttp://www.encolombia.com/odontologia/foc/foc20202-enfermedad.htm

9. Benítez Melo A.P, Romero Sánchez M.R. Gingivitis en niños y púberes tempranos pertenecientes a hogares de beneficio social de la sabana de Bogotá. Rev. Fed. Odontol. Colomb. 2005 nov. [Acceso: 15 de abril de 2010]; (24):[10-19]. Disponible en: http://bases.bireme.br/cgibin/wxislind. exe/iah/online/?!sisScript=iah/iah.xis\&src=google \&base=LILACS\&lang $=p \&$ extAction $=$ Ink\&exprS earch $=42$ | 897\&indexSearch=ID

I0. Boj R., Catala M., García-Ballesta C. Odontopediatría. 2da ed. Ed. Masson. Barcelona España. 2005. Cap. 32.

II. Marquillas Bras J., Pediatría en Atención Primaria. 2da ed. Ed. Masson. Barcelona España. 2005. Cap. 58.
12. Rodríguez V. J., Monzón J. E. Relación entre índice de sangrado papilar en la enfermedad periodontal en niños y la prevalencia de bacterias anaerobias y espiroquetas. Resumen: M-05I. UNIVERSIDAD NACIONAL DEL NORDESTE Comunicaciones Cientificas y Tecnologicas.2003 [Acceso: 27 de abril de 2010]. Disponible en: http://www.unne. edu.ar/Web/cyt/cyt/2003/comunicaciones/03Medicas/M-05I.pdf

13. Jahn, M. R, Jahn, R. S "Fique atento: criança também tem gengivite" Rev. Assoc. Paul. Cir. Dent. Jul.-ago.1997. [acceso: 15 de abril de 2010];5। (4): 355 - 8. Disponible en: http://bases.bireme. br/cgibin/wxislind.exe/iah/online/?lsisScript=iah/ iah. $x$ is \&src $=$ google \&base $=$ LILACS\&lang $=p \&$ next Action=Ink\&exprSearch=203086\&indexSearch= ID

14. Discacciati de Lértora M. S., Quintero de Lucas G. V: Guía de Trabajos Prácticos. Cátedra Odontopediatría. Aprobada por Resolución $\mathrm{N}^{\circ}$ 070/05. C. D. FOUNNE. Año 2005.

15. Dho S., Gili, A. Análisis de Salud Bucal en alumnos de una escuela periférica de la ciudad de Corrientes, Argentina. Revista de la Facultad de Odontología UNNE. 2009 [Acceso: 17de abril de 20I0];2 (I): 43 - 5I. Disponible en: http://odn. unne.edu.ar/volii_I.pdf.

16. Rodríguez-Carrasco, J., Rivera- Cuello, J.M, Salud bucodental en menores institucionalizados de Granada. Boletín SAPAO. 2009. [Acceso: II de noviembre de 20I0]3(3):74- 95. Disponible en: http://www.spao.info/Boletin/3_3/3_3_original. pdf

17. Lescano de Ferrer A. Estudio descriptivo de los tejidos gingivales en niños de dentición temporaria y mixta temprana. Claves Odontol. Mar.2007. [acceso: 17de abril de 2010]; 14(59): 9-16. Disponible en http://bases.bireme.br/cgibin/wxislind. exe/iah/online/?!sisScript=iah/iah.xis\&src=google \&base=LILACS\&lang=p\&nextAction $=$ Ink\&exprS earch $=498230$ \&indexSearch=ID.

18. Harris N.; García Godoy F. Odontología Preventiva Primaria $2^{\circ}$ Edición. Manual Moderno. 2005.

19. Pauritaite J., Milciuviene S. The prevalence of gingivitis among 4-16 year old school children in Kaunas. Stomatologija, Baltic Dental and Maxillofacial Journal 2003. [Acceso: 17de abril de 2010]; 5:97-100. Disponible en:http://www.sbdmj.com/033/033-04.pdf

20. Juárez - López, M. L., Pruneda J. F. Prevalencia y factores de riesgo asociados a enfermedad periodontal en preescolares de la Ciudad de México. Gac. Med. Mex. 2005 [Acceso: 19 de abril de 
2010] I4I(3): I85-I89. Disponible en: www.medigraphic.com/pdfs/gaceta/gm-2005/gm053c.pdf

21. Franco M. C. Prevalencia de Caries y Gingivitis en Preescolares. Rev. CES Odontología- 1995. [Acceso: 17de abril de 20I0].8 (2). I28-I3I.Disponible en: http://bases.bireme.br/cgibin/wxislind.exe/ iah/online/?lsisScript=iah/iah.xis\&src=google\&bas $\mathrm{e}=$ LILACS\&lang=p\&nextAction=Ink\&exprSearch $=515178$ \&indexSearch=ID.

22. González C., Navarro J. C. Cambio de indicadores de placa dento- bacteriana, gingivitis y caries dental en niños entre 2 y 5 años a partir de una intervención educativa dirigida a madres de preescolar. Rev.CES Odontología. Vol. 19. № I. 2006. [Acceso: 17de abril de 2010]; 19 (I): 9 17. Diponible en: http://www.imbiomed.com. $\mathrm{mx} / \mathrm{I} / \mathrm{l} /$ articulos.php? $\mathrm{method=}$ showDetail\&id articulo $=52397 \&$ id_seccion $=31 \mid 12 \& i d$ _ ejemplar $=5307 \&$ \&d_revista $=\mid 88$.

23. OPS - OMS "La salud de la niñez" 126.a SESIÓN DEL COMITÉ EJECUTIVO Washington, D.C.,26 al 30 de junio de 2000. Disponible en: http:// www.paho.org/spanish/gov/ce/cel26_16.pdf

24. OMS: La OMS publica un nuevo informe sobre el problema mundial de las enfermedades bucodentales. Disponible en: http://www.who.int/mediacentre/news/releases/2004/pr I5/es/index.

25. Science and Therapy Committee of the American Academy of Periodontology Academy Report: Epidemiology of Periodontal Diseases. J Periodontol. 2005 [Acceso: 20 de noviembre de 2010]; 76: I406 - 1419. Disponible en: http:// www.perio.org/resources-products/posppr3-3. html

26. Coutinho, T C; Lopes T.A. Prevalência de gengivite em crianças. RGO (Porto Alegre); 1997. [Acceso: I7de abril de 20I0] 45(3): I70-4, mayojun. Disponible en: http://bases.bireme.br/cgibin/ wxislind.exe/iah/online/?/sisScript=iah/iah.xis\&src =google\&base=BBO\&lang=p\&nextAction=Ink\&e xprSearch $=16415$ \&indexSearch=ID.

27. Varas F., Zillmann G., Muñoz A. Estado periodontal y necesidades de tratamiento en niños de 6 a 8 años de edad de la Región Metropolintana. Año 2004-2006. Rev. Soc. Chil. Odontopediatria. 2008. [Acceso: 19 de abril de 20l0]. 23 (2): 9. Disponible en: http://www.odontopediatria.cl/ Publicaciones/23-2/23_2.pdf.

28. Dhar V., Jain A. Prevalence of gingival diseases, malocclusion and flourosis in school-going children of rural areas in Udaipur district. Journal Indian Soc.Pedod. Prev.Dent. 2007. [Acceso: 19 de abril de 2010]. Apr-Jun; 25 (2): 103 - 5. Disponible en:www.ncbi.nlm.nih.gov/pubmed//7660647.

29. Vila, V. G., Romero H. Estudio del Estado Gingi- val relacionado con la higiene en adolescentes y adultos que asistieron a la Cátedra Practica Clínica Preventiva. Jornadas de Ciencia y Tecnología de la Facultad de Odontología. UNNE. [Acceso: 19 de abril de 2010]. Disponible en: www.odn. unne.edu.ar/2jcyt2009.pdf.

30. Ketabi, M, Tazhibi, M. The prevalence and Risk Factors of Gingivitis among the children Referred to Islamic Azad University (Khorasgan Branch) Dental School, in Iran.Dental Research Journal. 2006. [Acceso: II de noviembre de 2010] 3 (I): I- 4. Disponible en: http://www.sid.ir/en/ VEWSSID/J_pdf// I 4820060 I07.pdf.

3I. Murrieta Pruneda J. F., Juárez López L. A., Linares Vieyra C., Zurita Murillo V. Prevalencia de gingivitis en un grupo de escolares y su relación con el grado de higiene oral y el nivel de conocimientos sobre salud bucal demostrado por sus madres. Bol Méd Hosp Infant Méx 2004; Vol. 61 (I): 44 - 54.

32. Bustamante, Z, Camargo Cruz, L. Estado de Salud Bucal de niños preescolares de nivel socioeconómico alto y medio alto Medellin. 1997. CES ODONTOLOGÍA, 1998 [Acceso: II de abril de 20I0]; II,(I). Disponible en: http://bdigital.ces.edu.co/ojs/index.php/odontologia/rt/printerFriendly/84I/0.

33. Lindhe, Lang, Karring. Periodontologia Clinica e Implantologia Odontologica. Ed. Médica Panamericana. $5^{\circ}$ Ed. Madrid, España. 2008. Cap. 17. 